\title{
Effect of magnet position on tipping and bodily tooth movement in magnetic force-driven orthodontics
}

\section{Yoshiki Ishida}

Harvard School of Dental Medicine

\section{Yukinori Kuwajima}

Harvard School of Dental Medicine

\section{Cliff Lee}

University of California, San Francisco School of Dentistry

\section{Kaho Ogawa}

Harvard School of Dental Medicine

John Daren Da Silva

Harvard School of Dental Medicine

Shigemi Ishikawa-Nagai ( $\nabla$ shigemi_nagai@hsdm.harvard.edu )

Harvard School of Dental Medicine

\section{Research article}

Keywords: Magnets, CAD/CAM, Superimposition, Orthodontics, typodont, 3D printer, 3D scanner

Posted Date: December 30th, 2019

DOI: https://doi.org/10.21203/rs.2.19722/v1

License: (c) (i) This work is licensed under a Creative Commons Attribution 4.0 International License. Read Full License

Version of Record: A version of this preprint was published at Materials on August 13th, 2020. See the published version at https://doi.org/10.3390/ma13163588. 
$1<$ Title $>$

2 Effect of magnet position on tipping and bodily tooth movement in magnetic force-driven

3 orthodontics

4

$5<$ Authors $>$

6 Yoshiki Ishida ${ }^{1}$, Yukinori Kuwajima ${ }^{1}$, Cliff Lee $^{2}$, Kaho Ogawa ${ }^{1}$, John D. Da Silva ${ }^{3}$,

$7 \quad$ Shigemi Ishikawa-Nagai ${ }^{1}$

$8{ }^{1}$ Department of Oral Medicine, Immunity and Infection, Harvard School of Dental

9 Medicine, 188 Longwood Avenue, Boston, MA, United States

$10{ }^{2}$ Department of Orofacial Sciences, Division of Periodontology, University of California,

11 San Francisco School of Dentistry, 513 Parnassus Ave, San Francisco, CA, United States

$12{ }^{3}$ Department of Restorative Dentistry and Biomaterial Sciences, Harvard School of

13 Dental Medicine, 188 Longwood Avenue, Boston, MA, United State

$<$ Corresponding author $>$

Shigemi Ishikawa-Nagai, Yukinori Kuwajima 


\section{Abstract}

Magnets have many advantages for orthodontic applications. The purpose of this study was to investigate the effect of the magnet position on the tipping and bodily tooth movement using 3D digital analysis ex vivo.

Orthodontic typodont models for space-closing ( $2 \mathrm{~mm}$ diastema) were created, mimicking maxillary central incisors. Tooth models $(10 \mathrm{~mm}$ crown and $14 \mathrm{~mm}$ root length) created by $3 \mathrm{D}$ printer were used for the typodont model. Nd-Fe-B magnets were placed in the middle of the tooth (Model-M) and the cervical area (Model-C). The typodonts were immersed in a hot water-bath at $55^{\circ} \mathrm{C}$ to initiate tooth movement. The scanned typodont data before and after tooth movement were superimposed, and 3D coordinates $(\mathrm{X}, \mathrm{Y}, \mathrm{Z})$ on the tooth were obtained. The root apex data was also obtained by superimposing the designed tooth model on the crown portion of the typodont model. 3D movement of the crown and the root apex, moving speed, degree of rotation (yaw, pitch, and roll) in 2 models were analyzed and compared by Pearson's correlation confidents. Moderate crowding typodont cases were treated with two magnet position settings, and patterns of tooth/root movement and rotation were compared. 
37 root apex moved to the opposite direction of the crown movement with a negative

38 correlation. In contrast, the crown and root apex moved in the same direction with a

39 positive correlation in Model-C. In the ex vivo moderate crowding case, Model-C created

40 bodily movement, in which the cusp and root apex both moved in the same direction with

41 less tooth rotation. magnetic force was able to increase the amount of bodily tooth movement and decrease unwanted rotation/tipping in an ex vivo setting.

$<$ Keywords $>$ 


\section{Introduction}

Rare earth magnets, such as Sm-Co and Nd-Fe-B magnets were introduced in the 1970s and 1980s (1-3). Rare earth magnets have magnetic saturation, coercivity (resistance to demagnetization), and energy. These properties allowed for the production of small magnets. Nd-Fe-B magnets are less costly to produce than Sm-Co alloys, and are the most common rare earth permanent magnet in use today (3-6). Magnets have many advantages for orthodontic applications $(1,4,7,8)$. Magnets does not decrease orthodontic force during tooth movement. Magnets can exert forces for tooth movement through physical barriers, such as mucosa and bone, and do not require patient compliance, as is the case with elastic bands. Oral hygiene may be performed more easily than with conventional fixed orthodontics with auxiliaries such as hooks, elastics, and spring coils. The magnetic force produced is inversely proportional to the square of the distance between them $(1,8-10)$. Magnets have been used in orthodontic treatment with limited approaches. The first report of the use of magnetic force to move teeth was in 1977 when Kawata and Takeda (11) described a technique of using magnetic brackets of $\mathrm{Co}-\mathrm{Cr}-\mathrm{Fe}$ alloy, bonded to the upper anterior and the lower anterior teeth, for the closure of interdental spaces $(1,3,4)$. Prasad et al. reported that Nd-Fe-B magnets 
and clinical reports using Nd-Fe-B magnets in orthodontic treatment, such as for forced eruption of impacted teeth $(4,5,8,10,12-14)$. From an esthetic standpoint, magnets may not be ideal due to the black or metallic color. However, FeCo-(Al-fluoride) nanogranular films exhibiting ferromagnetic properties with high optical transparency in the visible light spectrum have been recently introduced (15).

The ultimate goal is the establishment of a magnetic force-driven orthodontics as a future treatment modality in comprehensive orthodontic care. The first analysis the efficacy of $\mathrm{Nd}-\mathrm{Fe}-\mathrm{B}$ magnetic attraction and repulsion forces by means of a three dimensional (3D) digital analysis of movement (distance, direction, angulation and duration) and rotation (yaw, pitch and roll) of the crown and root of teeth in an ex vivo typodont model (16). We found that magnets are able to achieve desired tooth movements in a space-closing model, space-gain model and a moderate crowding case in an ex-vivo setting. However, more tipping movement and rotation were observed when using attractive forces. Bodily tooth movement more ideal that tipping tooth movement, as tipping may destructively influence the periodontal tissue in the cervical area (17). It was also shown that hyalinization occurs less frequently during bodily tooth movement than during tipping movements, because forces are more evenly distributed along the root surface during bodily movement (18). In this study, we hypothesized that the magnet 
91 position can contribute to avoiding the occurrence of tipping movements. The purpose of

92 this study was to clarify the effect of the magnet position on the tipping and bodily tooth

93 movement by means of 3D digital analysis of tooth movement in $\mathrm{X}, \mathrm{Y}$ and $\mathrm{Z}$ axis, and

94 rotation (yaw, pitch and roll) ex vivo.

95 Materials and Methods

\section{Fabrication of typodont model with magnets and 3D digital scanning.}

Dental typodonts mimicking maxillary central incisors were used in this ex vivo

experiment. Dental typodont models of maxillary central incisors $(24 \mathrm{~mm}$ length, $10 \mathrm{~mm}$

width, and $8.0 \mathrm{~mm}$ crown thickness) were designed using CAD software (Creo

Elements/Direct Modeling Express 4.0, PTC, Needham, MA, USA). Three $1.5 \mathrm{~mm}$

diameter x $1.0 \mathrm{~mm}$ height cone-shaped measurement horns were created on the center of

the incisal edge, distal and lingual side of the tooth (Figure 1a). The designs were exported

as STL files. The tooth models were fabricated using a 3D printer (MiiCraft 125, MiiCraft

Inc., Hsinchu, Taiwan) using model resin (Next Dent Model 2.0, Next Dent BV,

Soesterberg, Netherland). 
typodont boxes (Figure 1b) in order to accurately superimpose data. Typodont boxes were fabricated in the same manner as the tooth models.

Ni-plated cylindrical Nd-Fe-B N52 magnets (NeoMag Co., Ltd., Chiba, Japan, $2.0 \mathrm{~mm}$ diameter x $5.0 \mathrm{~mm}$, surface magnetic flux density $414 \mathrm{mT}$, adsorption power 22 $\mathrm{kPa}$, density $7.5 \mathrm{~g} / \mathrm{cm}^{3}$ ) were used in this ex vivo study. The magnets were bonded to the tooth with the north pole of the maxillary left central incisor (UL1) magnet facing the mesial and the south pole of the maxillary right central incisor (UR1) magnet facing the mesial, to produce attraction force, and the teeth were placed $2 \mathrm{~mm}$ apart. Two settings of magnet position were tested in this study. The magnet was placed in the middle of tooth crown (Model-M) and in the cervical area (Model-C, Figure 1b). The teeth were then stabilized in the typodont box using paraffin wax (Paraffin wax, GC Co., Ltd., Tokyo, Japan, solidification point $59.3^{\circ} \mathrm{C}$ ). A plastic guide made with self-curing acrylic resin (Pattern Resin, GC Chicago, IL, USA) to standardize tooth position in each of 30 typodonts.

The typodonts were scanned using a 3D laser scanner (Ortho Insight 3D Laser Scanner, Motion View LLC., Chattanooga, TN, USA) prior to tooth movement. The typodonts were positioned in the center of the scanning table with the magnet facing forward. After the initial scan, the typodonts were divided into 10 groups, with 3 
127 typodonts in each group for a total of 30 typodonts. The typodonts were immersed in a

digital thermostatic water bath (Joan Lab Digital Thermostatic Water Bath Manufacturer, Ningbo Yinzhou Joan Lab Equipment Co., Ltd., Zhejiang, China, 3 L capacity) at $55^{\circ} \mathrm{C}$ to initiate tooth movement from $5 \mathrm{~min}$ and up to $50 \mathrm{~min}$ in $5 \mathrm{~min}$ increments depending on the group. After complete immersion of the typodont model in the water bath for the prescribed time, the models were stabilized in a cold-water bath at $5^{\circ} \mathrm{C}$ for $30 \mathrm{~min}$. After stabilization the models were dried and scanned in the same manner as the initial scans.

The scanned typodont model data was converted into STL files using 3D visualization software (Ortho Insight 3D, Motion View LLC., Chattanooga, TN, USA). The pre-movement STL files were superimposed on the post-movement using 3D data inspection software (GOM inspect, GOM, Braunschweig, Germany) using the 112 landmarks on the typodont box. 3D coordinates (X, Y, Z axis) of each of the 3 measurement points on the tooth model of UR1 and UL1 were obtained (Figure 2a). 3D coordinates (X, Y, Z axis) of the center of root apex (CRA, Figure 2b) of UR1 and UL1 were obtained by superimposing designed tooth model on the crown portion of typodont model.

\section{Analysis of the movement and rotation of the tooth crown portion.}

The center of gravity (CG) of each tooth crown was calculated using 3D 
145 coordinates of 3 measurement points by the following equation (1).

$146 \mathrm{CG}(\mathrm{x})=\frac{X_{1}+X_{2}+X_{3}}{3} \quad \mathrm{CG}(\mathrm{y})=\frac{Y_{1}+Y_{2}+Y_{3}}{3} \quad \mathrm{CG}(\mathrm{z})=\frac{Z_{1}+Z_{2}+Z_{3}}{3}$

147 The amount of 3D movement of the CG of each tooth crown portion (ACG) in each of 10

148 time-series was calculated by the following equation (2):

$149 \operatorname{ACG}(X)=C G(X)_{\text {post }}-C G(X)_{\text {pre }}$

$150 A C G(Y)=C G(Y)_{\text {post }}-C G(Y)_{\text {pre }}$

151

$A C G(Z)=C G(Z)_{\text {post }}-C G(Z)_{\text {pre }}$

152 The speed of $\mathrm{X}$-axis movement $(\mathrm{mm} / \mathrm{min})$ was calculated, using ACG by the following 153 equation (3):

$154 \operatorname{Speed}(\mathrm{mm} / \mathrm{min})=\frac{A C G_{(t)}-A C G_{(t-5)}}{5}$

155 where $A C G_{(t)}$ is the amount of $3 \mathrm{D}$ movement of $\mathrm{CG}$ in the duration of $t$ minutes. The average movement speed on Model-M and Model-C were compared. two cartesian coordinates, we can calculate the rotation using the dot and cross of the two vectors using equations $(4,5)$. The cross product will be used as the normal axis (n), and 160 dot product will show the angle $(\theta)$ of the rotation.

$161 n=a \times b$ 
163 From the angle and the axis, it is possible to construct the rotation matrix (R) by using

164 Rodrigues' rotation formula (19) (6) below.$$
\left[\begin{array}{ccc}
\cos \theta+n_{x}^{2}(1-\cos \theta) & n_{x} n_{y}(1-\cos \theta)-n_{z} \sin \theta & n_{x} n_{z}(1-\cos \theta)+n_{y} \sin \theta \\
n_{y} n_{x}(1-\cos \theta)+n_{z} \sin \theta & \cos \theta+n_{y}^{2}(1-\cos \theta) & n_{y} n_{z}(1-\cos \theta)-n_{x} \sin \theta \\
n_{z} n_{x}(1-\cos \theta)-n_{y} \sin \theta & n_{z} n_{y}(1-\cos \theta)+n_{x} \sin \theta & \cos \theta+n_{z}^{2}(1-\cos \theta)
\end{array}\right]
$$

168 There are several ways to show the rotational transition, we use the yaw (Z-axis), pitch (Xaxis), and roll (Y-axis) angles.

$$
\left[\begin{array}{ccc}
\cos \beta \cos \alpha & \sin \gamma \sin \beta \cos \alpha-\cos \gamma \sin \alpha & \sin \gamma \sin \alpha+\cos \gamma \sin \beta \cos \alpha \\
\cos \beta \sin \alpha & \sin \gamma \sin \beta \sin \alpha+\cos \gamma \cos \alpha & -\sin \gamma \cos \alpha+\cos \gamma \sin \beta \sin \alpha \\
-\sin \beta & \sin \gamma \cos \beta & \cos \gamma \cos \beta
\end{array}\right]
$$

171 Given the rotation matrix (7), we can derive gamma, beta, alpha from the following

172 equation (8):

$R_{31}=-\sin \beta$

$174 \quad R_{32}=\sin \gamma \cos \beta$

$R_{33}=\cos \gamma \cos \beta$

\section{Analysis of the movement of center of root apex.}

$A C R(X)=C R A(X)_{\text {post }}-C R A(X)_{\text {pre }}$

$$
A C R(Y)=C R A(Y)_{\text {post }}-C R A(Y)_{\text {pre }}
$$


$A C R(Z)=C R A(Z)_{\text {post }}-C R A(Z)_{\text {pre }}$

\section{Application of the magnetic force-driven technique to the moderate crowding case.}

A moderate crowding case including teeth maxillary right canine (UR3) to maxillary left canine (UL3) was created in the typodont model and the magnets were placed to setup both attraction and repulsion forces to straighten the arch form (Figure 4). The attraction magnet force was placed in 5 areas (UR4 mesial - UR3 distal, UR2 mesial - UR1 distal, UR1 mesial - UL1 mesial, UL1 distal - UL2 mesial, UL3 distal - UL4 mesial) and repulsion force was placed in 2 areas (UR3 mesial - UR2 distal and UL2 distal - UL3 mesial). A nickel-titanium archwire, 0.012 inch Sentalloy (TOMY INTERNATIONAL INC., Tokyo, Japan) was used as to guide the movement and establish the desired arch form. The model was scanned pre and post-movement and 3D movement and rotation were analyzed in the same manner.

\section{Statistical analysis}

Crown and root movement, crown speed, and crown rotation in each of 2 groups were obtained and averaged in each of 10 time-series and average data was used for the statistical analysis. Two-way ANOVA and Pearson's correlation coefficient were used $(\mathrm{p}<0.01)$.

\section{Results}




\section{Crown movement}

201 in all three axes $(\mathrm{p}<0.01)$ (Figure 5). Cervical placement of the magnet resulted in less

202 movement, in the $\mathrm{X}$ and $\mathrm{Z}$ axes, with no difference in the $\mathrm{Y}$ axis. The largest movement

203 in three dimensions was X-axis on both models, which was the intended movement. The

204 amount of movement on the X-axis in 50 minutes on Model-M and Model-C at $1.01 \mathrm{~mm}$

205 and $0.64 \mathrm{~mm}$, respectively.

206 2. Speed

Magnet position influenced the speed of movement, with the maximum rate of movement on Model-M and Model-C at $0.050 \mathrm{~mm} / \mathrm{min}$ and $0.043 \mathrm{~mm} / \mathrm{min}$, respectively.

The greatest movement speed was observed on the X-axis, which was the intended direction of movement (Figure 6).

\section{Tooth rotation}

Magnet position and duration were both significant factors on yaw and roll

213 (Figure 7). The largest rotation was observed on yaw in both Model-M and Model-C at

2143.22 degrees and 1.39 degrees, respectively, in 50 minutes $(p<0.01)$. In the pitch, there

215 were no significant differences between Model-M and Model-C. When considering roll, 
$217(p<0.01)$, at 2.89 degrees and 0.67 degrees, respectively $(\mathrm{p}<0.01)$.

\section{Relationship (Association) of tooth and root movement}

Magnet position was a significant factor in the direction of root apex movement

(Figure 8). In Model-M, ACR moved in the opposite direction to ACG, but in Model-C,

221 ACG and ACR moved in the same direction. In Model-M, a weak negative correlation was observed on Z-axis $(R=0.78)$ (Figure $9 \mathrm{~d}-\mathrm{f})$.

5. Application of the magnetic force-driven technique to the moderate crowding case. observed, in which the root apex moved in the opposite direction of the cusp. In contrast, in Model-C, bodily movement was observed, in which the cusp and root apex both moved distally. Less tooth rotation was observed in in Model-C compared to Model-M.

Discussion maximum rate of tooth movement with minimal irreversible damage to the root, 
235 periodontal ligament, and alveolar bone (20). The level of optimal force for orthodontic

236 tooth movement is controversial and has not yet been defined (21), as it is a consequence

237 of multiple biological responses to orthodontic force. Higher forces do not always move

238 teeth faster than lower forces, but higher forces had more areas of hyalinization (22).

239 Recent studies have indicated that continuous forces result in faster tooth

240 movement than intermittent forces (23-25), but continuous forces caused greater root

241 resorption with a greater unwanted rotational movement. Root-resorption and alveolar

242 bone resorption are major biological damage observed by inappropriate orthodontics

243 applications (23-26). Unwanted rotational movement results in more root resorption in

244 the middle third level of the tooth (23). It can be summarized that the magnitude of

245 optimal force should be determined individually for each patient and intermittent force

246 should be applied to minimize unwanted outcomes by monitoring tooth movement

247 closely. Accurate force control is an essential factor.

Different types of loading force produce different types of tooth movement (27).

249 Cervical bone resorption and total alveolar bone thickness at mid-root and apical levels

250 were decreased with tipping movements compared to bodily movement $(17,28)$.

251 Different settings of auxiliary appliances can create the different movements, such as a 
28). In our previous study, attractive magnet force applied in the middle of maxillary

central incisor for $2 \mathrm{~mm}$ diastema closure in ex vivo model created the tipping movement

(16). Tipping movement was identified by 3D digital assessment of movement and

rotation of tooth and root apex. In this study, we assessed tooth and root apex movement and rotation with 2 different setting of the position of magnet placement, one at the middle of crown (Model-M) and the other in the cervical area (Model-C).

As we hypothesized, the position of the magnet was a significant factor for creating tipping or bodily movement. Bodily movement is produced with linear force, but

261 tipping forces are produced with lever force with a fulcrum, which in the case of orthodontics is the level of the alveolar bone, or in the case of this study is the level of the paraffin wax. By moving the force more incisally, the distance of the lever force to the fulcrum increases, which for the same amount of force would produce a higher proportion of torque/rotation force along the fulcrum. By moving the force more apically, the opposite occurs, decreasing the amount of tipping force and increasing the linear force for bodily movement. This was confirmed with the 3D digital analysis which indicated that the Model-C created more bodily movement than Model-M, which had greater movement/speed due to the increased force output with a lever, but created more unwanted tipping/rotation. This result was same as the report by Lee et al. (29). The use 
271 of greater magnet force at the cervical area can compensate for the decrease of tooth

272 movement distance and speed while minimizing unwanted rotation. The ex vivo moderate

273 crowding case validated that Model-C created bodily movement and less rotation on the

274 canines with attracting force.

Placing orthodontic brackets in conventional fixed orthodontics at the cervical aspect of the tooth crown creates unwanted side effects due to the proximity to the

277 gingival margin, with gingival enlargement/hyperplasia and gingivitis being the most common (30). This is likely due to a combination of difficult oral hygiene leading to plaque accumulation, as well as interactions between the metal from the brackets and the periodontium. Magnets could offer a significant advantage in this regard, with a much smaller profile without additional hooks or coiled springs allowing for oral hygiene and minimizing plaque accumulation. setting. One drawback to the ex vivo environment is the lack of interaction of oral fluids on the magnet which may potentially create corrosion. However, new coatings have been developed to combat corrosion, such as nickel/alumina composite coatings and multilayer titanium nitride ceramic coatings, as well as the development of corrosion resistant magnets, such as iron-platinum (FePt) magnets (31). Tooth movement in vivo is also 
290 typodont model and further research is required to study the clinical environment.

\section{Conclusion}

292 The $3 \mathrm{D}$ data obtained in this study validated that modifying the position of the

293 applied magnetic force was able to increase the amount of bodily tooth movement and 294 decrease unwanted rotation/tipping in an ex vivo setting. Magnets were able to achieve 295 desired bodily tooth movements in a moderate crowding ex vivo model with an attractive 296 force.

\section{List of abbreviations}

298 3D Three dimensional

299 UL1 Maxillary left central incisor

$300 \quad$ UR1 Maxillary right central incisor

301 Model-A Model with the magnet placed in the middle of tooth crown

302 Model-C Model with the magnet placed in the cervical area

303 CRA Center of root apex

304 CG Center of gravity

$305 \quad$ ACG

Amount of 3D movement of the center of gravity

306 ACR

Amount of 3D movement of the center of root apex 
308 UR2 Maxillary right lateral incisor

309 UL3 Maxillary left canine

310 UR3 Maxillary right canine

311 UL4 Maxillary left first premolar

312 UR4 Maxillary right first premolar

313 Ethics approval and consent to participate

314 Not applicable.

315 Consent for publication

316 Not applicable.

317 Availability of data and materials

318 The dataset used and/or analyzed during the current study are available from the

319 corresponding author on reasonable request.

320 Competing interests

321 Not applicable.

$322 \quad$ Funding

323 There was no funding support available for this research.

324 Authors' contributions 
325 Our study was carried out with collaboration of all authors. SIN conceived and designed

326 the study. YI, YK and KO performed the experiments and analyzed the data. CL and JDD

327 interpreted the results. YI, CL, JDD and SIN wrote the manuscript. All authors approved

328 the final manuscript.

329 Acknowledgements

330 Not applicable.

$331 \quad$ References

332 1. Darendeliler MA, Darendeliler A, Mandurino M. Clinical application of magnets

333 in orthodontics and biological implications: a review. European journal of orthodontics.

334 1997;19(4):431-42.

335 2. Robinson AL, Gschneidner K, Capellen JJ-THYoRE. Powerful new magnet 336 material found. North-Holland Publishing Co., Amsterdam; 1987. p. 19.

337 3. Bhat VS, Shenoy KK, Premkumar PJAoM, Sciences H. Magnets in dentistry. $338 \quad 2013 ; 1(1): 73$.

339 4. Prasad M, Manoj-Kumar M, Gowri-Sankar S, Chaitanya N, Vivek-Reddy G, 340 Venkatesh N. Clinical evaluation of neodymium-iron-boron (Ne2Fe14B) rare earth 341 magnets in the treatment of mid line diastemas. Journal of clinical and experimental 342 dentistry. 2016;8(2):e164-71. 
3435 Hwang HS, Lee KH. Intrusion of overerupted molars by corticotomy and 344 magnets. American journal of orthodontics and dentofacial orthopedics : official 345 publication of the American Association of Orthodontists, its constituent societies, and 346 the American Board of Orthodontics. 2001;120(2):209-16.

347 6. Riley MA, Walmsley AD, Harris IR. Magnets in prosthetic dentistry. The Journal 348 of prosthetic dentistry. $2001 ; 86(2): 137-42$.

3497 Muller M. The use of magnets in orthodontics: an alternative means to produce 350 tooth movement. European journal of orthodontics. 1984;6(4):247-53.

351 8. Huang TTY, Elekdag-Turk S, Dalci O, Almuzian M, Karadeniz EI, Gonzales C, 352 et al. The extent of root resorption and tooth movement following the application of 353 ascending and descending magnetic forces: a prospective split mouth, microcomputed354 tomography study. European journal of orthodontics. 2017;39(5):547-53.

3559 9. Mussard M, Le Mouël J-L, Poirier J-PJCRG. On some early attempts to 356 determine the variation of the magnetic force with distance. 2013;345(5-6):225-9.

357 10. Bondemark L, Kurol J, Hallonsten AL, Andreasen JO. Attractive magnets for 358 orthodontic extrusion of crown-root fractured teeth. American journal of orthodontics and 359 dentofacial orthopedics : official publication of the American Association of 360 Orthodontists, its constituent societies, and the American Board of Orthodontics. 
1997;112(2):187-93.

362

11. Kawata T, Takeda S, editors. NEW ORTHODONTIC APPLIANCE BY MEANS

OF MAGNETIC BRACKET. Journal of Dental Research; 1977: AMER ASSOC

DENTAL RESEARCH 1619 DUKE ST, ALEXANDRIA, VA 22314.

12. Sandler JP. An attractive solution to unerupted teeth. American journal of orthodontics and dentofacial orthopedics : official publication of the American Association of Orthodontists, its constituent societies, and the American Board of

13. Phelan A, Tarraf NE, Taylor P, Honscheid R, Drescher D, Baccetti T, et al.

Skeletal and dental outcomes of a new magnetic functional appliance, the Sydney

Magnoglide, in Class II correction. American journal of orthodontics and dentofacial

orthopedics : official publication of the American Association of Orthodontists, its

14. Zhao N, Feng J, Hu Z, Chen R, Shen G. Effects of a novel magnetic orthopedic appliance (MOA-III) on the dentofacial complex in mild to moderate skeletal class III children. Head \& face medicine. 2015;11:34.

Ferromagnetic Nanogranular Films with Tunable Transmittance. Scientific reports. 
380 16. Kuwajima Y, Ishida Y, Lee C, Mayama H, Satoh K, Ishikawa-Nagai SJH. 3D

381 digital analysis of magnetic force-driven orthodontic tooth movement. $382 \quad 2019 ; 5(11): \mathrm{e} 02861$.

383 17. Kondo T, Hotokezaka H, Hamanaka R, Hashimoto M, Nakano-Tajima T, Arita

$384 \mathrm{~K}$, et al. Types of tooth movement, bodily or tipping, do not affect the displacement of the 385 tooth's center of resistance but do affect the alveolar bone resorption. The Angle 386 orthodontist. 2017;87(4):563-9.

387 18. Pilon JJ, Kuijpers-Jagtman AM, Maltha JC. Magnitude of orthodontic forces and 388 rate of bodily tooth movement. An experimental study. American journal of orthodontics 389 and dentofacial orthopedics : official publication of the American Association of 390 Orthodontists, its constituent societies, and the American Board of Orthodontics. $391 \quad 1996 ; 110(1): 16-23$.

392 19. Olinde R. Des lois gometriques qui regissent les dplacements d'un systme solide dans l'espace, et de la variation des coordonnes provenant de ces dplacement consideres 394 ind; pendent des causes qui peuvent les produire. J Math Pures Appl 1840;5:380-400. 20. Burstone CJJTbotm. The biophysics of bone remodeling during orthodontics 396 (optimal force considerations). 1989:321-33. 
397 21. Ren Y, Maltha JC, Kuijpers-Jagtman AM. Optimum force magnitude for 398 orthodontic tooth movement: a systematic literature review. The Angle orthodontist. $399 \quad 2003 ; 73(1): 86-92$.

400 22. Ren Y, Maltha JC, Van 't Hof MA, Kuijpers-Jagtman AM. Optimum force 401 magnitude for orthodontic tooth movement: a mathematic model. American journal of 402 orthodontics and dentofacial orthopedics : official publication of the American 403 Association of Orthodontists, its constituent societies, and the American Board of 404 Orthodontics. 2004;125(1):71-7.

405 23. Ozkalayci N, Karadeniz EI, Elekdag-Turk S, Turk T, Cheng LL, Darendeliler 406 MA. Effect of continuous versus intermittent orthodontic forces on root resorption: A 407 microcomputed tomography study. The Angle orthodontist. 2018;88(6):733-9.

408 24. Ballard DJ, Jones AS, Petocz P, Darendeliler MA. Physical properties of root 409 cementum: part 11. Continuous vs intermittent controlled orthodontic forces on root 410 resorption. A microcomputed-tomography study. American journal of orthodontics and 411 dentofacial orthopedics : official publication of the American Association of 412 Orthodontists, its constituent societies, and the American Board of Orthodontics. $413 \quad 2009 ; 136(1): 8 . e 1-8 ;$ discussion -9.

414 25. Maltha JC, van Leeuwen EJ, Dijkman GE, Kuijpers-Jagtman AM. Incidence and 
415 severity of root resorption in orthodontically moved premolars in dogs. Orthodontics \&

416 craniofacial research. 2004;7(2):115-21.

417 26. Gay G, Ravera S, Castroflorio T, Garino F, Rossini G, Parrini S, et al. Root 418 resorption during orthodontic treatment with Invisalign $\AA$ : a radiometric study. $419 \quad 2017 ; 18(1): 12$.

27. Kusy RP, Tulloch JF. Analysis of moment/force ratios in the mechanics of tooth

421 movement. American journal of orthodontics and dentofacial orthopedics : official 422 publication of the American Association of Orthodontists, its constituent societies, and 423 the American Board of Orthodontics. 1986;90(2):127-31.

424 28. Chaimongkol P, Thongudomporn U, Lindauer SJ. Alveolar bone response to 425 light-force tipping and bodily movement in maxillary incisor advancement: A prospective 426 randomized clinical trial. The Angle orthodontist. 2018;88(1):58-66.

427 29. Lee BW. The force requirements for tooth movement, Part I: Tipping and bodily 428 movement. Australian orthodontic journal. 1995;13(4):238-48.

429 30. Gursoy UK, Sokucu O, Uitto VJ, Aydin A, Demirer S, Toker H, et al. The role of 430 nickel accumulation and epithelial cell proliferation in orthodontic treatment-induced 431 gingival overgrowth. European journal of orthodontics. 2007;29(6):555-8.

432 31. Yiu EY, Fang DT, Chu FC, Chow TW. Corrosion resistance of iron-platinum 
magnets. Journal of dentistry. 2004;32(6):423-9.

434

\section{Legends}

Figure 1. Typodont model used in this study. (a) design and sizes of maxillary left central incisor (UL1), (b) scheme of typodont model.

Figure 2. Explanations of tooth movements and rotations. (a) X, Y and Z axis direction of tooth movement, (b) Identification of center or root apex.

Figure 3. Explanations of tooth rotations. (a) yaw, (b) pitch, (c)roll.

Figure 4. Typodont model of a moderate crowding case including teeth UL3-UR3. Canine is supposed to move distally by magnetic force-driven.

Figure 5. Average amount of movement on the center of gravity (ACG). (a) X-axis, (b) Y-axis, (c) Z-axis.

Figure 6. Average moving speed of the center of gravity.

446 Figure 7. Average amount of tooth rotation. (a) yaw, (b) pitch, (c) roll.

447 Figure 8.3D graphs of amount of movement of the center of gravity of each tooth crown portion (ACG) and the center of root apex (ACR). (a) Model-M, (b) Model-C. 
Figure 9. Average amount of 3D movement of the center of gravity of each tooth crown portion (ACG) and the center of root apex (ACR). (a) X-axis, (b) Y-axis, (c) Z-axis on Model-M, and (d) X-axis, (e) Y-axis, (f) Z-axis on Model-C. moderate crowding case. (a) Images of UR3 and UL3 of pre- and post-movement, (b) distance moved on Crown and root apex, (c) mesial tooth rotation. 


\section{Figures}

(a)

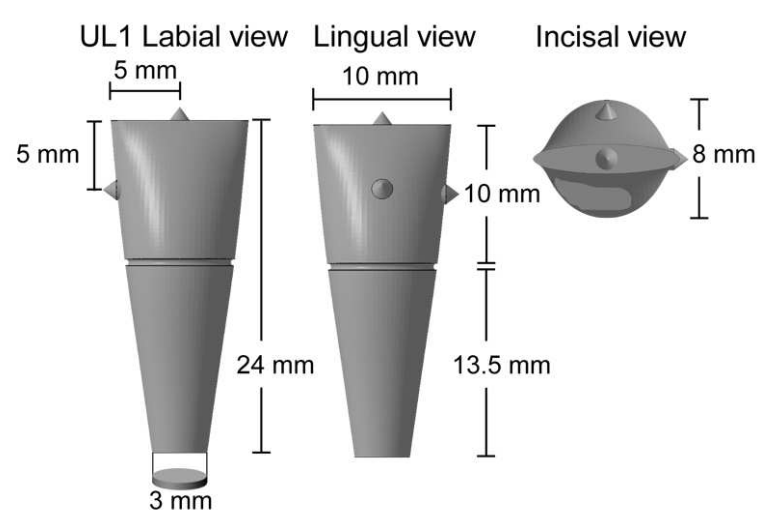

(b)

Model-M
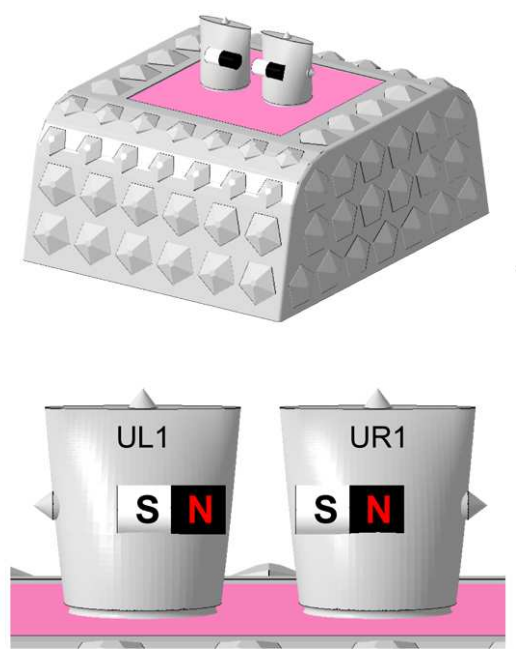
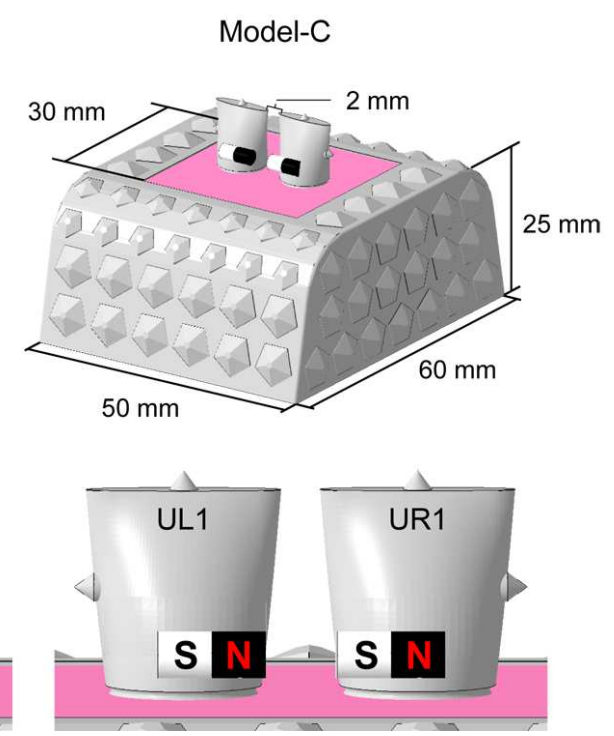

Figure 1

Typodont model used in this study. (a) design and sizes of maxillary left central incisor (UL1), (b) scheme of typodont model.

(a)

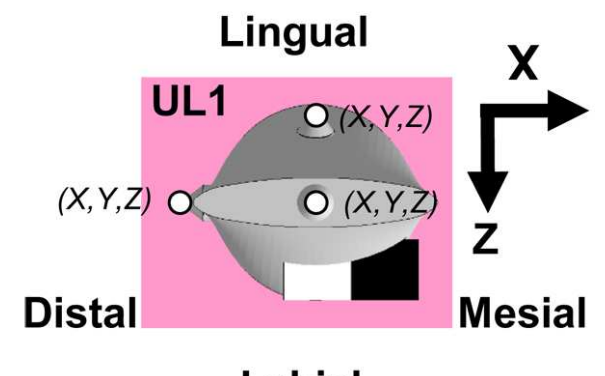

\section{Labial}

Labial view

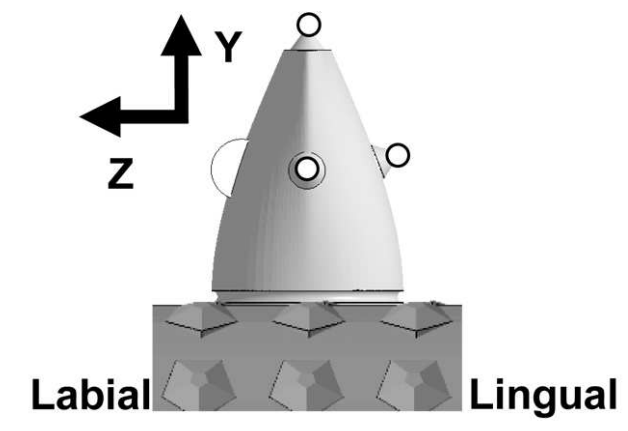

Distal view (b)

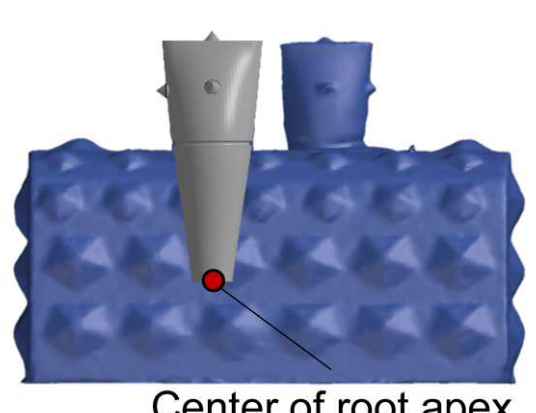

Center of root apex

Figure 2

Explanations of tooth movements and rotations. (a) $X, Y$ and $Z$ axis direction of tooth movement, (b) Identification of center or root apex. 
(a)
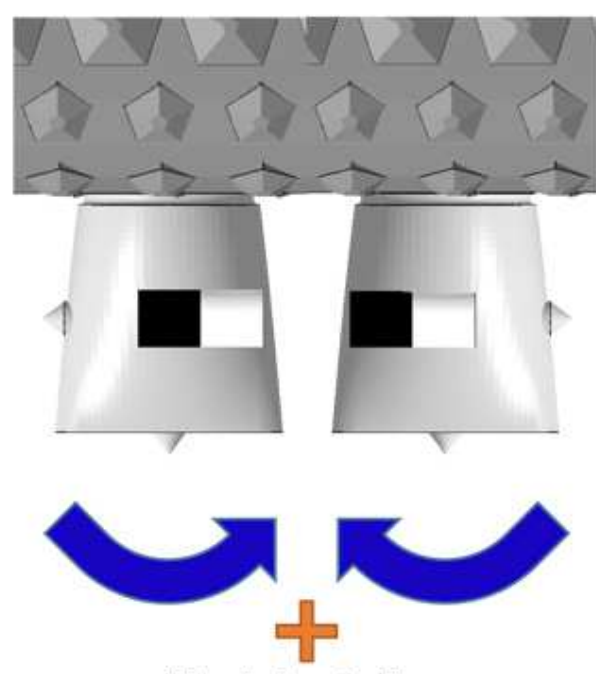

Mesial rotation

Labial view (b)
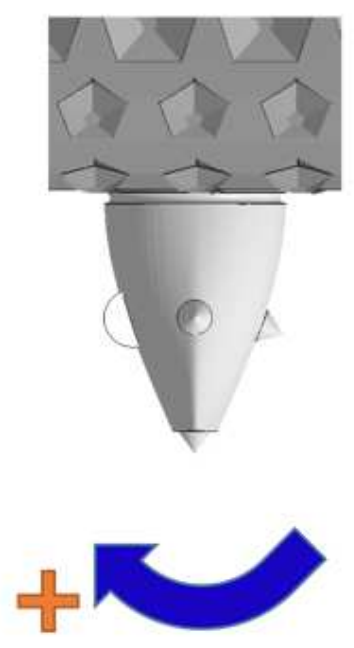

Labial-Inclination

Distal view (c)

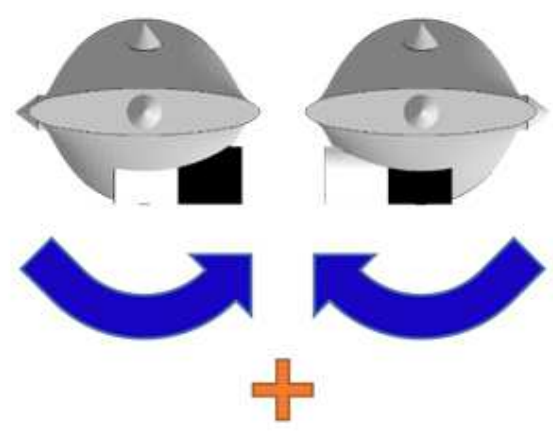

Mesial rotation Incisal view

Figure 3

Explanations of tooth rotations. (a) yaw, (b) pitch, (c)roll. 


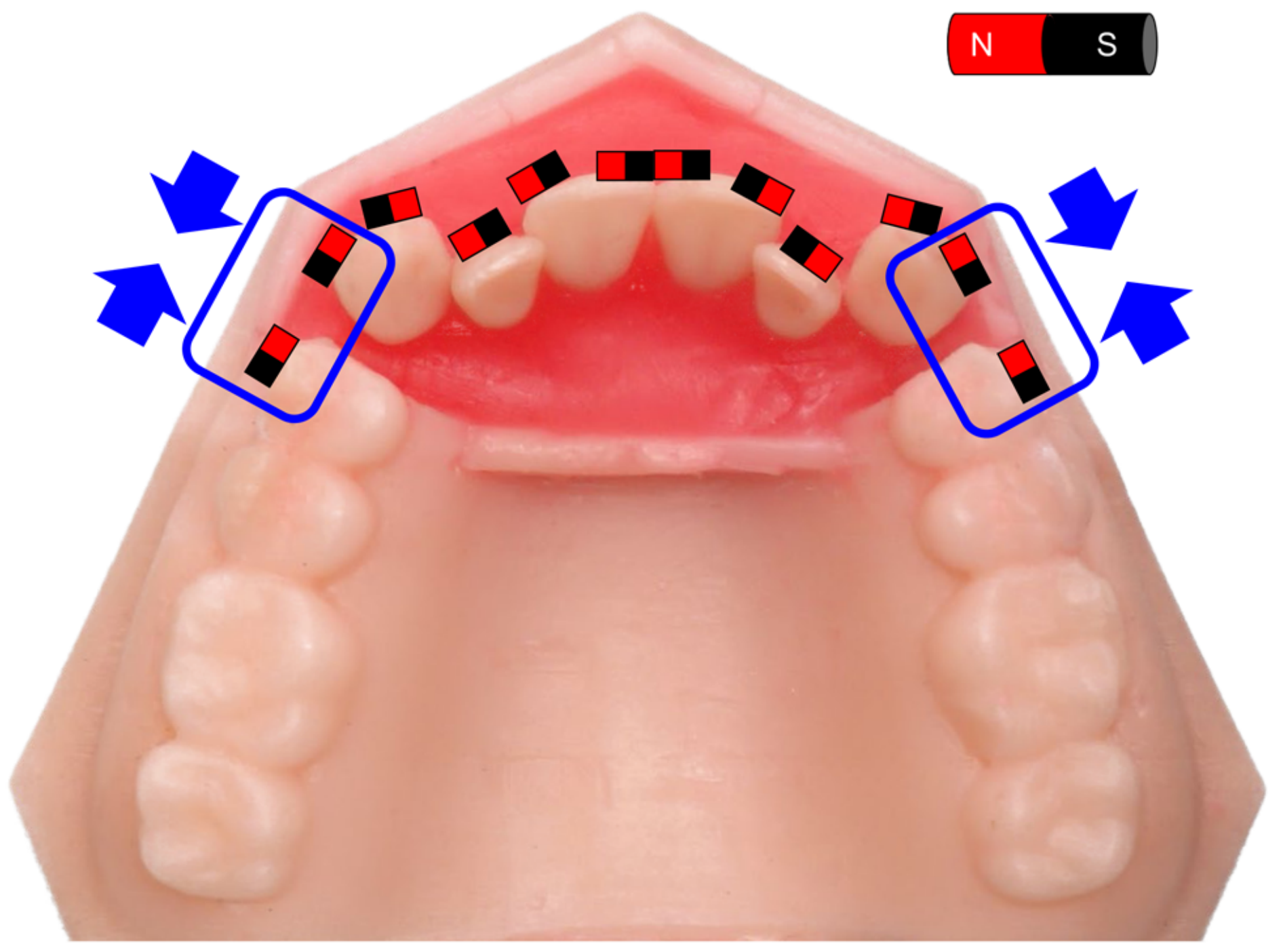

\section{Figure 4}

Typodont model of a moderate crowding case including teeth UL3-UR3. Canine is supposed to move distally by magnetic force-driven.
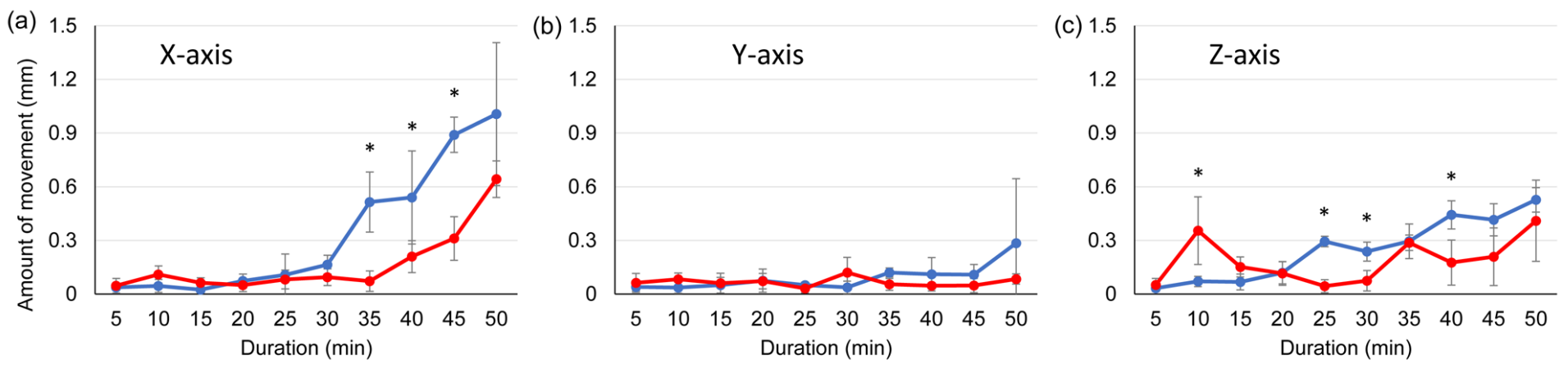

$\bigcirc$ Model-M $\bigcirc$ Model-C $\quad *$ : Significant differences between Model A and Model B $(p<0.01)$

\section{Figure 5}

Average amount of movement on the center of gravity (ACG). (a) X-axis, (b) Y-axis, (c) Z-axis. 

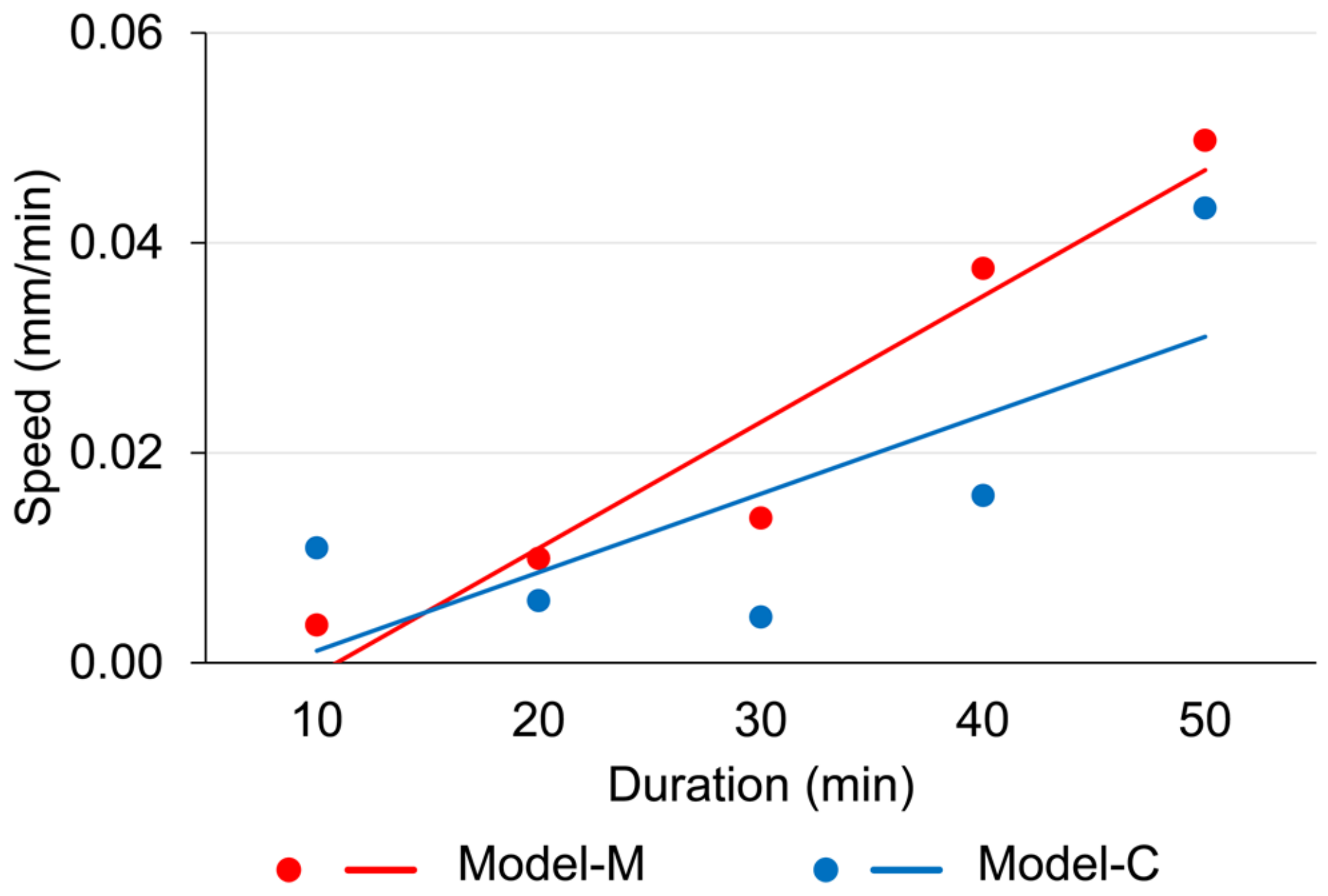

Figure 6

Average moving speed of the center of gravity.
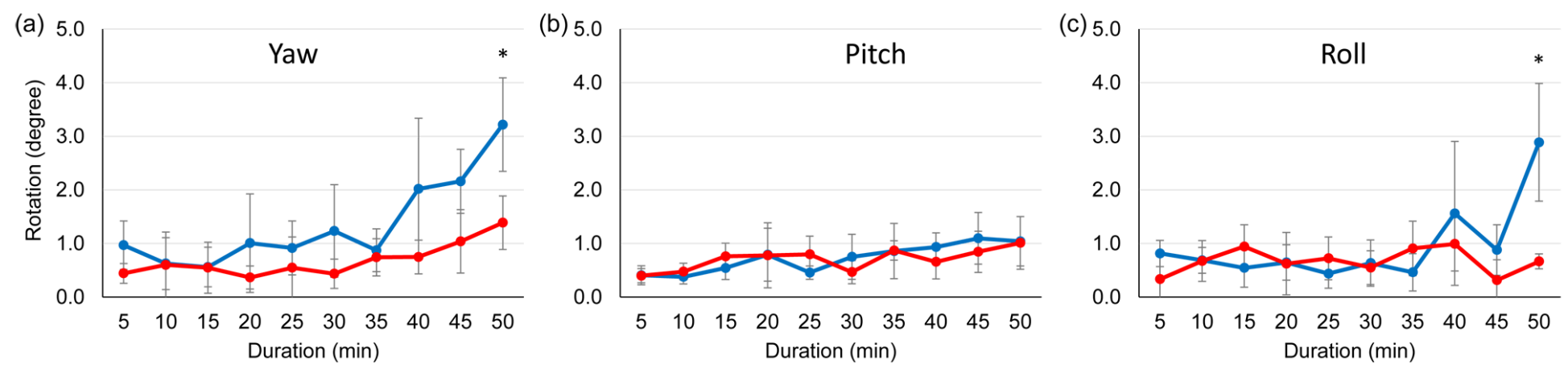

- Model-M $\quad$ Model-C * : Significant differences between Model-M and Model C $(p<0.01)$

Figure 7

Average amount of tooth rotation. (a) yaw, (b) pitch, (c) roll. 

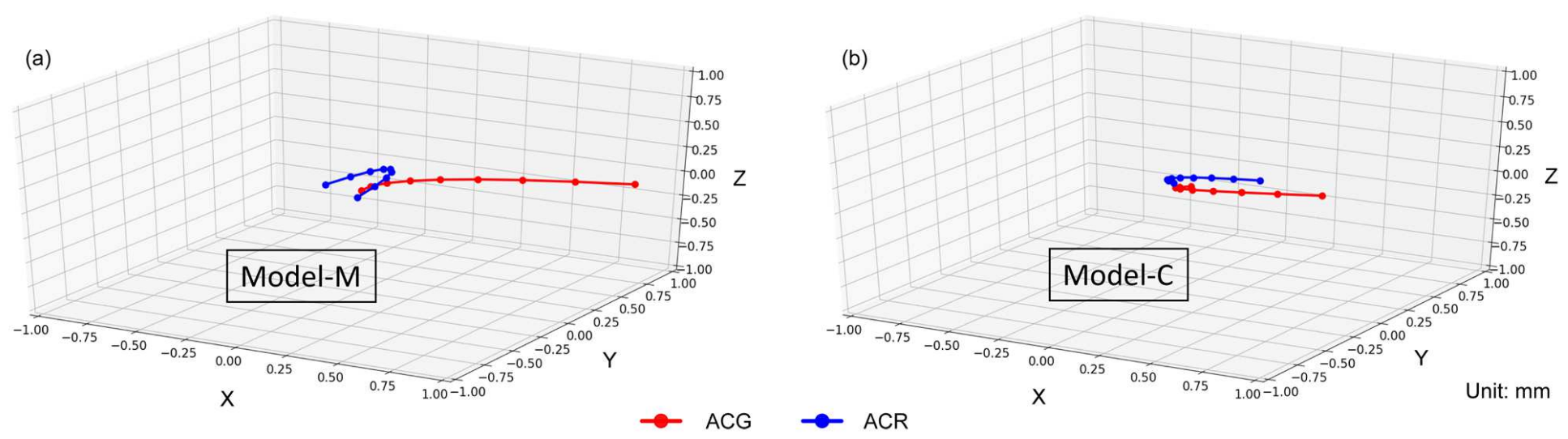

Figure 8

3D graphs of amount of movement of the center of gravity of each tooth crown portion (ACG) and the center of root apex (ACR). (a) Model-M, (b) Model-C.

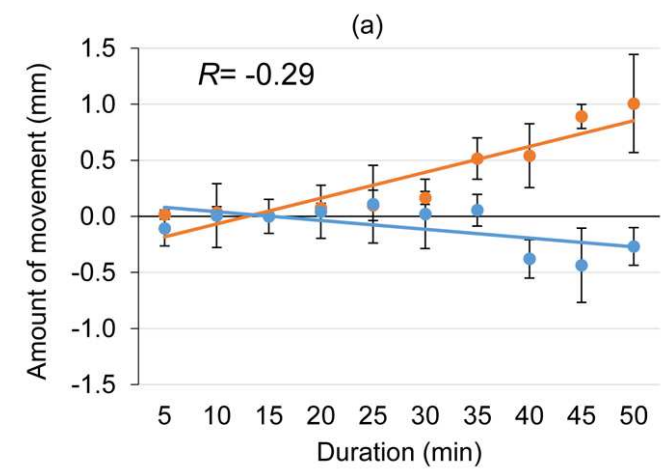

(d)

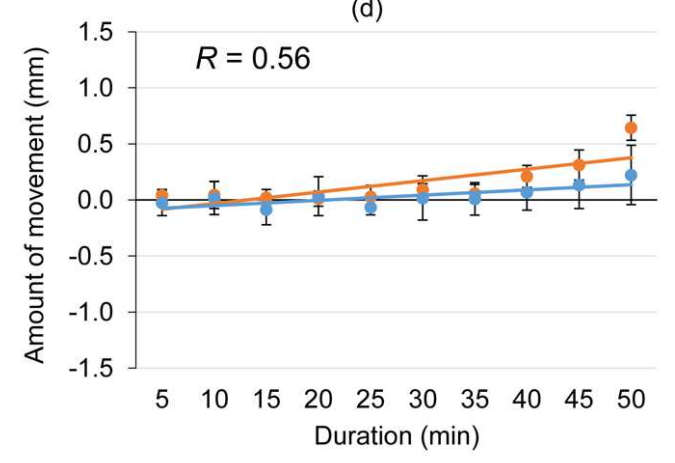

O ACG O ACR

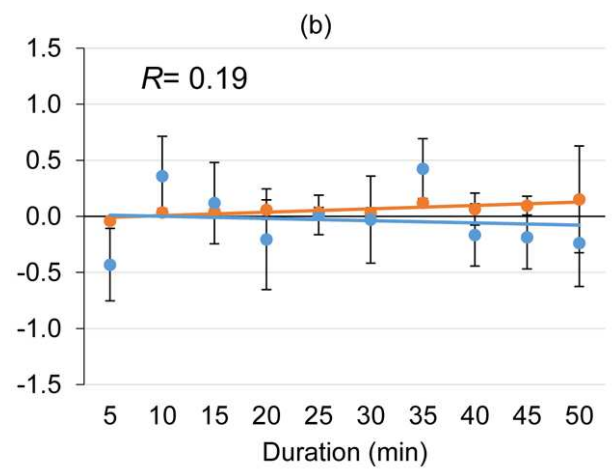

(e)

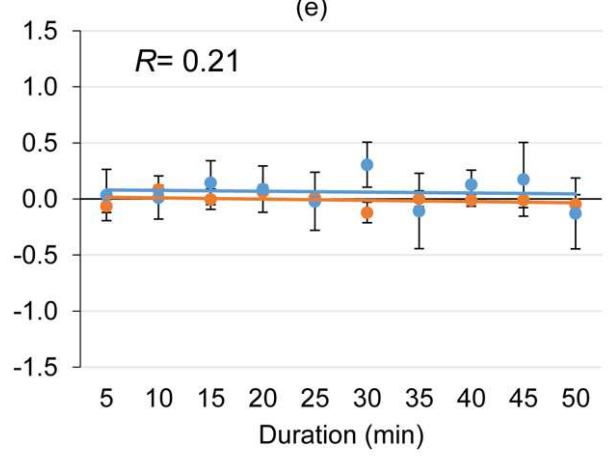

$R$ : Pearson's correlation coefficient between ACG and ACR

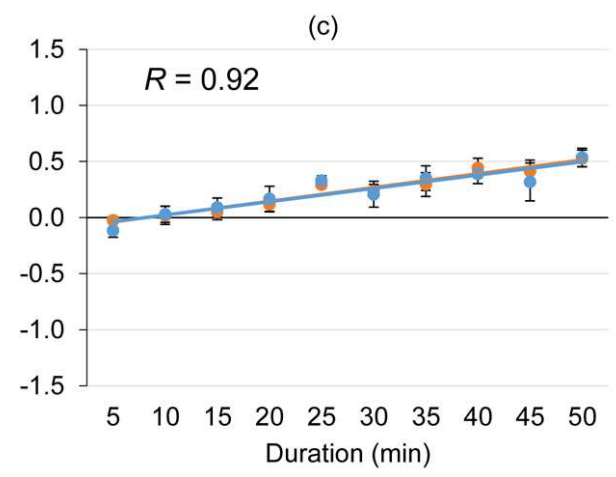

(f)

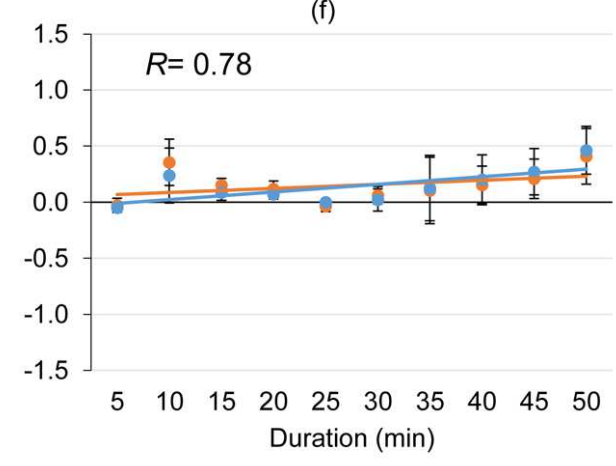

Figure 9

Average amount of 3D movement of the center of gravity of each tooth crown portion (ACG) and the center of root apex (ACR). (a) X-axis, (b) Y-axis, (c) Z-axis on Model-M, and (d) X-axis, (e) Y-axis, (f) Z-axis on Model-C. 


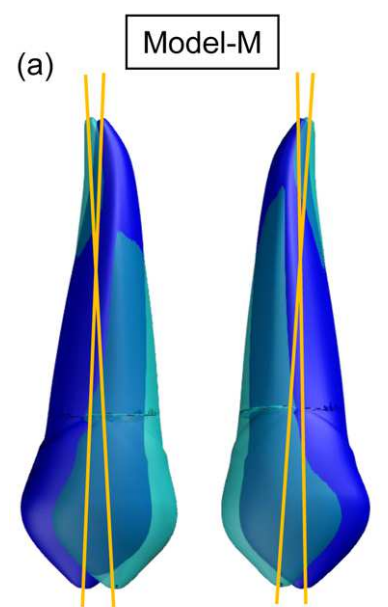

$\begin{array}{cc}\text { UR3 } & \text { UL3 } \\ \text { Pre-movement } \\ \text { Post-movement }\end{array}$

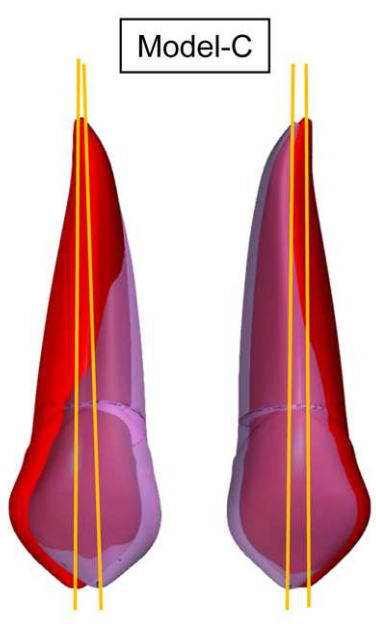

UL3
Pre-movement

Post-movement (b)

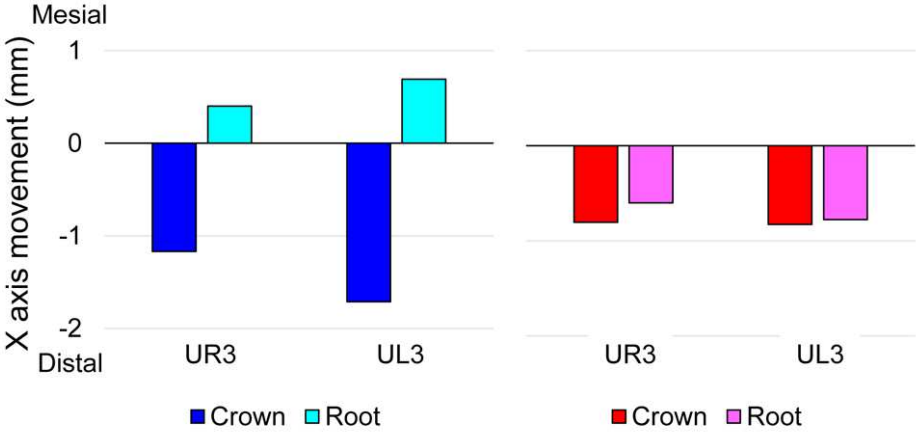

(c)

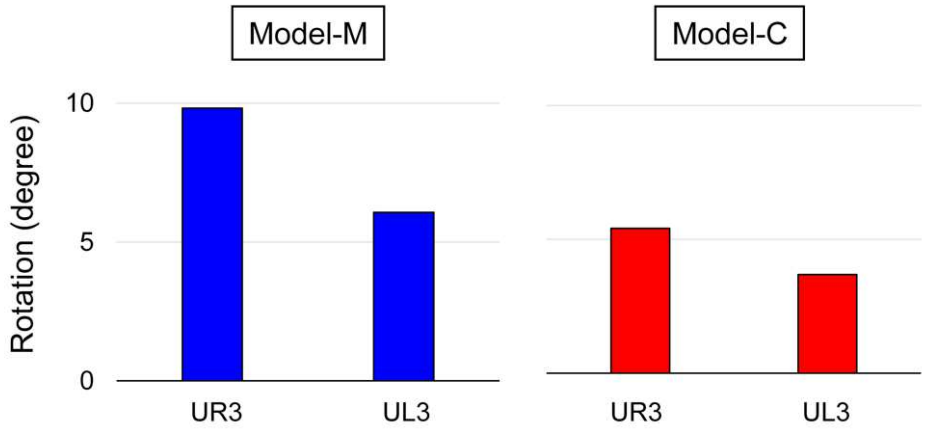

Figure 10

Results of tooth movement by magnetic force-driven on typodont model of a moderate crowding case. (a) Images of UR3 and UL3 of pre- and post-movement, (b) distance moved on Crown and root apex, (c) mesial tooth rotation. 